University of Wollongong

Research Online

Faculty of Engineering and Information

Faculty of Engineering and Information

Sciences - Papers: Part B

Sciences

2018

\title{
Antibacterial and Antifungal Activity of Poly(Lactic Acid)-Bovine Lactoferrin Nanofiber Membranes
}

\author{
Raul Machado \\ University of Minho \\ André Da Costa \\ University of Minho \\ A C. Gomes \\ University of Minho \\ Margarida Casal \\ University of Minho
}

Dina M. Morais da Silva

University of Wollongong

See next page for additional authors

Follow this and additional works at: https://ro.uow.edu.au/eispapers1

Part of the Engineering Commons, and the Science and Technology Studies Commons

Research Online is the open access institutional repository for the University of Wollongong. For further information contact the UOW Library: research-pubs@uow.edu.au 


\title{
Antibacterial and Antifungal Activity of Poly(Lactic Acid)-Bovine Lactoferrin Nanofiber Membranes
}

\author{
Abstract \\ Antimicrobial materials have become relevant for local therapies preventing microbial resistance induced \\ by systemic antibiotic treatments. This work reports the development of electrospun poly(lactic acid) \\ (PLLA) nanofiber membranes loaded with bovine lactoferrin (bLF) up to $20 \mathrm{wt} \%$. The membranes present \\ smooth and nondefective fibers with mean diameters between $717 \pm 197$ and $495 \pm 127 \mathrm{~nm}$, and an \\ overall porosity of $\approx 80 \%$. The hydrophobicity of the PLLA membranes is reduced by the presence of bLF. \\ The release profile of bLF correlates with an anomalous transport model, with $17.7 \pm 3.6 \%$ being released \\ over 7 weeks. The nanofiber mats show no cytotoxicity on human skin fibroblasts and even promote cell \\ proliferation after short exposure periods. Furthermore, the developed membranes display antifungal \\ activity against Aspergillus nidulans by inhibiting spore germination and mycelial growth. These results \\ evidence the strong potential of bLF-PLLA nanofiber membranes to be used as antifungal dressings. \\ Disciplines \\ Engineering | Science and Technology Studies

\section{Publication Details} \\ Machado, R., da Costa, A., Silva, D. M., Gomes, A. C., Casal, M. \& Sencadas, V. (2018). Antibacterial and \\ Antifungal Activity of Poly(Lactic Acid)-Bovine Lactoferrin Nanofiber Membranes. Macromolecular \\ Bioscience, 18 (3), 1700324-1-1700324-10.

\section{Authors} \\ Raul Machado, André Da Costa, Dina M. Morais da Silva, A C. Gomes, Margarida Casal, and Vitor \\ Sencadas
}




\section{Full Paper}

\section{Antibacterial and antifungal activity of poly(lactic acid)-bovine lactoferrin nanofiber membranes ${ }^{\mathrm{a}}$}

Raul Machado*, André da Costa, Dina M. Silva, Andreia C. Gomes, Margarida Casal and Vitor Sencadas*

Dr. R. Machado, Dr. A. da Costa

CBMA (Centre of Molecular and Environmental Biology)

Department of Biology, University of Minho, Campus de Gualtar, 4710-057 Braga, Portugal

E-mail: raulmachado@bio.uminho.pt

Dr. D. M. Silva

School of Mechanical, Materials, Mechatronics and Biomedical Engineering

University of Wollongong, Wollongong, NSW 2522, Australia

Dr. A. C. Gomes, Prof. M. Casal

CBMA (Centre of Molecular and Environmental Biology)

Department of Biology, University of Minho, Campus de Gualtar, 4710-057 Braga, Portugal

Dr. Vitor Sencadas

School of Mechanical, Materials, Mechatronics and Biomedical Engineering

University of Wollongong, Wollongong, NSW 2522, Australia

ARC Center of Excellence for Electromaterials Science, University of Wollongong, 2522 NSW, Australia

E-mail: victors@uow.edu.au

\footnotetext{
a Supporting Information is available online from the Wiley Online Library or from the author.
} 
Antimicrobial materials have become a relevant for local therapies preventing microbial resistance induced by systemic antibiotic treatments. This work reports the development of electrospun PLLA nanofiber membranes loaded with bovine lactoferrin (bLF) up to $20 \mathrm{wt} \%$. The membranes presented smooth and non-defective fibers with mean diameters between 717 \pm 197 and $495 \pm 127 \mathrm{~nm}$, and an overall porosity of $\approx 80 \%$. The hydrophobicity of the PLLA membranes was reduced by the presence of bLF. The release profile of bLF correlates with an anomalous transport model, with $17.7 \pm 3.6 \%$ being released over 7 weeks. The nanofiber mats showed no cytotoxicity on human skin fibroblasts and even promoted cell proliferation after short exposure periods. Furthermore, the developed membranes displayed antifungal activity against $\mathrm{A}$. nidulans by inhibiting spore germination and mycelial growth. These results evidence the strong potential of bLF-PLLA nanofiber membranes to be used as antifungal dressings.

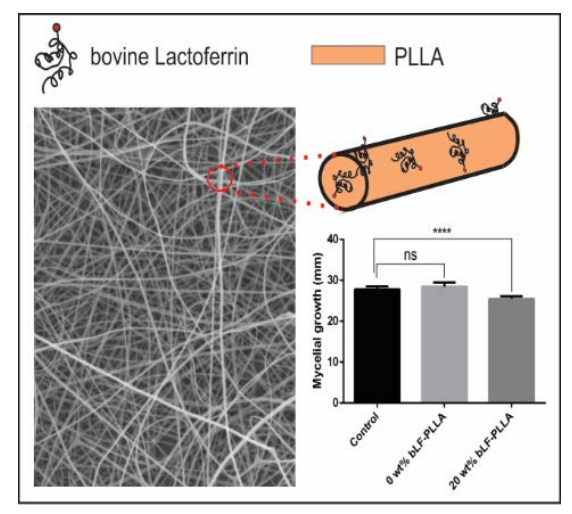




\section{Introduction}

The high incidence of infection by antibiotic-resistant bacteria is a growing health concern worldwide. In Europe alone, it is estimated to cause 25000 fatalities each year accounting for 1.5 billion euros spent annually ${ }^{[1]}$. Therefore, novel strategies to manage infection and avoid emergence of resistance during therapy, mainly through prophylactic approaches using antimicrobial coatings or dressings in medical devices ${ }^{[2]}$. For instance, topical antimicrobial therapy may be especially helpful in the treatment of skin wounds, where a local approach would be more effective in avoiding healing delays or progression to a systemic infection ${ }^{[3,4]}$. Ionic silver is the most studied antimicrobial agent with a far lower propensity for resistance development than antibiotics ${ }^{[5]}$. Currently, silver-based formulations are used to inhibit bacterial growth in several medical applications, such as functionalization of catheters, dental practice, coating of medical devices, in cosmetics and as wound dressings for open infected wounds, skin ulcers, compound fractures, and burn injuries ${ }^{[5-7]}$. However, the possible side effects related to long-term exposure to silver nanoparticles ${ }^{[8-11]}$ justify the quest for new biocompatible molecules that can be metabolized, thus avoiding cytotoxicity ${ }^{[12]}$.

Lactoferrin (LF), an $80 \mathrm{kDa}$ iron-binding glycoprotein of the transferrin family, is a major component of milk and can be found in the secondary granules of neutrophils, in mucosal surfaces and in biological fluids of different mammals, playing an important role in the innate immune response ${ }^{[12-14]}$. Amongst its physiological roles, including iron homeostasis, immune response, antioxidant, anticancer and anti-inflammatory properties, the antimicrobial activity is the most studied ${ }^{[14-16]}$. LF is active against a broad spectrum of Gram-positive ${ }^{[17,18]}$ and Gram-negative ${ }^{[19,20]}$ bacteria, fungi ${ }^{[21,22]}$, viruses ${ }^{[23,24]}$ and protozoa ${ }^{[25]}$. This activity is based on the ability to sequester iron, an essential nutrient for pathogens, or by direct interaction with the microorganisms ${ }^{[26]}$; thus, structural integrity under processing is essential for keeping its bioactivity. 
Electrospinning is a versatile and inexpensive polymer processing technique used to produce continuous nanostructured fibrous materials ${ }^{[27]}$. This approach allows the processing of many polymers as well as composites for a wide set of applications, including biomedical scaffolds ${ }^{[28]}$ which may have incorporated functional proteins or peptides ${ }^{[29]}$ for additional functions. Poly(lactic acid) (PLLA) is a bio-based, biodegradable polymer derived from renewable resources such as starch from corn or potato ${ }^{[30]}$. Being classified as GRAS (Generally Recognized As Safe) by the FDA (Food and Drug Administration), this is a very attractive polymer for the pharmaceutical and biomedical industries due to its biocompatibility and biodegradability ${ }^{[31]}$. Additionally, PLLA is easily processed by several techniques, including electrospinning, enabling the tuning of its physico-chemical and biological properties ${ }^{\text {[32-35] }}$. In view of the above mentioned, the aim of this work was to evaluate the feasibility of developing composite PLLA-bovine lactoferrin (bLF) membranes by electrospinning, as a promising new alternative to conventional antimicrobial wound dressings. The processing effects were assessed by a thorough physical-chemical characterization of the composites with the bLF release profile also being evaluated. The cytotoxicity of the electrospun fiber mats was assessed using human fibroblasts and the antimicrobial activity evaluated against Pseudomonas aeruginosa, Staphylococcus aureus and Aspergillus nidulans.

\section{Experimental Section}

\subsection{Materials}

Purasorb PL18 (PLLA), with an average molecular weight of 217,000 - 225,000 g.mol ${ }^{-1}$, was purchased from Purac, bovine lactoferrin (bLF) was obtained from DMV International (USA) with a reported composition of $96 \mathrm{wt} \%$ dry weigh of protein, approximately $120 \mathrm{ppm}$ of iron, 0.5 wt\% of ash and 3.5 wt\% of moisture. Dimethylformamide (DMF) and dichloromethane 
(DCM) of analytical grade were purchased from Sigma-Aldrich. All materials and chemicals were used as received.

\subsection{Solution Preparation}

PLLA solution was prepared according to the method reported elsewhere ${ }^{[35]}$. For the composite samples, PLLA at a concentration of $10 \mathrm{wt} \%$ was dissolved in DMF/DCM (30/70, $\mathrm{v} / \mathrm{v}$ ) mixture, and the desired amount of bLF (0,10 and $20 \mathrm{wt} \%$, related to the polymer content) was added to the solution and placed in an ultrasound bath for $60 \mathrm{~min}$ for better dispersion of protein in the polymer solution.

\subsection{Electrospinning}

The polymer solution was transferred to a plastic syringe fitted with a steel needle with a diameter of $0.5 \mathrm{~mm}$. Electrospinning was conducted with an electric field between 0.75 and $1.5 \mathrm{kV} . \mathrm{cm}^{-1}$, applied with a high voltage power supply from Gamma High Voltage Research. A syringe pump (KDS 100L Pump from KDScientific) was used to feed the polymer solutions into the needle tip at a rate of $0.5 \mathrm{ml} . \mathrm{h}^{-1}$. The electrospun fibers were collected in grounded collecting plates (random fibers) placed at $15 \mathrm{~cm}$ from the needle.

\subsection{Characterization of the Nanofiber Membranes}

Samples were coated with a thin gold layer using a sputter coating (Smart Coater, JEOL) and their morphology was analyzed using a scanning electron microscopy (JEOL JSM-6490LV) with an accelerating voltage of $5 \mathrm{kV}$. The nanofibers average diameter and their distribution was calculated with at least 50 randomly selected fibers from SEM micrographs $(5,000 \mathrm{X}$ magnification), using ImageJ image processing software ${ }^{[36]}$.

Infrared measurements (FTIR) were performed at room temperature in a Spectrum Two ${ }^{\mathrm{TM}}$ IR spectrometer (Perkin Elmer) in ATR mode (UATR accessory, Perkin Elmer). Spectra were 
collected after 64 scans with a resolution of $4 \mathrm{~cm}^{-1}$ from 4000 to $400 \mathrm{~cm}^{-1}$. Normalization and spectra representation were performed with OriginPro 9.0 (OriginLab, Northampton, MA). The thermal behavior of the electrospun fiber mats was analyzed by differential scanning calorimetry measurements (DSC) with a TA Q100 apparatus (TA instruments). The samples were cut into small pieces from the middle region of the electrospun membranes and placed into $30 \mu \mathrm{l}$ aluminum pans and heated between 30 and $200{ }^{\circ} \mathrm{C}$ at a heating rate of $10{ }^{\circ} \mathrm{C} \cdot \mathrm{min}^{-1}$. All experiments were performed under a nitrogen purge. The thermal degradation kinetics was characterized by means of thermogravimetric analysis (TGA) in a TA Q500 (TA instruments), using different heating rates $\left(10,20,30\right.$ and $\left.40{ }^{\circ} \mathrm{C} \cdot \mathrm{min}^{-1}\right)$. All experiments were performed under argon atmosphere.

Water contact angle measurements (WCA, sessile drop in dynamic mode) were performed at room temperature in a Data Physics OCA20 device using ultrapure water as test liquid. The contact angles were measured by depositing water drops $(3 \mu \mathrm{L})$ on sample surface and analyzed with SCA20 software. At least 6 measurements were performed for each sample and in different locations, and the average contact angle was taken as the result for each sample.

Overall membrane porosity was determined by the pycnometer method following the procedure described elsewhere ${ }^{[37,38]}$. Briefly, the weight of the pycnometer filled with ethanol was measured and labeled as $W_{1}$; the sample with weight $W_{s}$ was immersed in ethanol. Subsequently, the sample was saturated by ethanol; additional ethanol was added to complete the volume of the pycnometer. Then, the pycnometer was weighted and labeled as $W_{2}$; the sample filled with ethanol was taken out of the pycnometer and the residual weight of the ethanol and the pycnometer was labeled as $W_{3}$. The porosity of the membrane was calculated according to Equation 1. The mean porosity of each membrane was obtained as the average of the values determined in three samples. 
$\varepsilon=\frac{W_{2}-W_{3}-W_{s}}{W_{1}-W_{3}}$

\subsection{In Vitro Release of Lactoferrin and Hydrolytic Degradation}

The in vitro release profile of the lactoferrin from $20 \mathrm{wt} \%$ bLF-PLLA membranes was determined under sink conditions. Membrane disks $(\varnothing=19 \mathrm{~mm})$ were weighted $\left(M_{1}\right)$ and placed in vessels containing $5 \mathrm{ml}$ of PBS buffer $\left(8\right.$ g.L ${ }^{-1} \mathrm{NaCl}, 0.2$ g. $\mathrm{L}^{-1} \mathrm{KCl}, 1.44$ g.L $\mathrm{L}^{-1}$ $\mathrm{Na}_{2} \mathrm{HPO}_{4}, 0.24$ g.L $\mathrm{L}^{-1} \mathrm{KH}_{2} \mathrm{PO}_{4}, \mathrm{pH} 7.4$ ) and incubated at $37^{\circ} \mathrm{C}$. At predetermined time points, the disks were removed, rinsed with $\mathrm{ddH}_{2} \mathrm{O}$ to remove the salts, and then dried under low pressure in a vacuum chamber (VACUO-TEMP, JP Selecta, Spain). After final weight determination $\left(M_{2}\right)$, hydrolytic degradation was calculated by Equation 2. The release medium was measured at $\lambda_{\max } 280 \mathrm{~nm}$ using a UV-Vis spectrophotometer (Shimadzu UV1800, Japan). Protein concentration was calculated using a standard curve (Equation 3) of bLF in PBS (pH 7.4). The release profile of bLF was calculated according to Equation 4.

$$
\begin{aligned}
& \operatorname{Mass} \operatorname{loss}(\%)=\left(1-\frac{M_{2}}{M_{1}}\right) \times 100 \\
& O D_{280 \mathrm{~nm}}=0.9778 x+0.01399\left(R^{2}=0.998\right)
\end{aligned}
$$

$b L F$ release $(\%)=\frac{\text { amount of } b L F \text { released at time } t}{\text { theoretical amount of bLF in the membrane }} \times 100$

\subsection{Release Kinetics Models}


bLF release results were fitted to the mathematical models to further study the protein release kinetics from the composite membranes. The best fit was achieved by the Korsmeyer-Peppas model (Equation 5):

$$
\frac{M_{t}}{M_{\infty}}=K t^{n}
$$

Where $\frac{M_{t}}{M_{\infty}}$ is a fraction of drug released at time $t, K$ is the rate constant, and $n$ is the exponent that characterizes the release mechanism ${ }^{[39,40]}$.

\subsection{Membrane Sterilization for Biological Assays}

The membranes were cut either in disks (antibacterial assays) or squares (antifungal assays) of adequate diameters and sterilized by UV exposure $(\lambda=254 \mathrm{~nm})$ for 30 minutes $(15$ minutes each side).

\subsection{Cell Culture and Cytotoxicity Evaluation}

Telomerase-immortalized normal human skin fibroblasts (BJ-5ta cell line) were obtained from the American Type Culture Collection (ATCC) through LGC standards and cultured in humidified environment at $37{ }^{\circ} \mathrm{C}, 5 \% \mathrm{CO}_{2}$, according to ATCC recommendations (BJ-5ta medium - 4 parts of Dulbecco’s modified Eagle’s medium containing 4 mM L-glutamine, 4.5 g/L glucose, 1.5 g/L sodium bicarbonate, and 1 part of Medium 199, supplemented with $10 \%$ (v/v) of foetal bovine serum (FBS), $1 \%$ (v/v) penicillin/streptomycin solution and $10 \mu \mathrm{g} / \mathrm{mL}$ hygromycin B).

Cell viability in response to short-term contact with the bLF-PLLA composites was assessed by MTS assay (CellTiter 96® Aqueous One Solution Cell Proliferation, Promega) according to manufacturer's instructions. UV sterilized samples of $100 \mathrm{~mm}^{2}$ were incubated with $750 \mu 1$ of cell culture medium without FBS for $24 \mathrm{~h}$ at $37{ }^{\circ} \mathrm{C}, 5 \% \mathrm{CO}_{2}$ in humidified environment. 
At the same time, $100 \mu \mathrm{l}$ of BJ-5ta cell suspension $\left(6.6 \times 10^{4}\right.$ cells $\left./ \mathrm{mL}\right)$ were seeded and cultured in surface treated 96-well plates (Nunclon polystyrene 96-well MicroWell, Thermo Scientific) for $24 \mathrm{~h}$ according to the cell culture conditions described above. After the incubation time, the cell culture medium was removed and replaced with the medium conditioned by contact with the bLF-PLLA membranes. Cells were incubated for an additional 24 and $72 \mathrm{~h}$ at $37^{\circ} \mathrm{C}, 5 \% \mathrm{CO}_{2}$, in humidified environment after which cell viability was measured using the MTS proliferation assay. Briefly, cells in $1 \mathrm{x}$ sterile PBS were incubated with MTS solution for $2 \mathrm{~h}$ at $37^{\circ} \mathrm{C}$ followed by reading the absorbance at $490 \mathrm{~nm}$ using standard PBS as blank measurement. Live cells react with tetrazolium salt in the MTS reagent producing a soluble formazan dye, which has absorbance at a wavelength of $490 \mathrm{~nm}$. In the linear range of the absorbance curve, the absorbance intensity is proportional to the number of metabolically active cells. Cells cultured in standard culture and in $30 \%$ DMSO were used as positive and negative controls for cell viability, respectively. Results were expressed as percentage of viability related to the positive control (set as $100 \%$ viability). All the experiments were performed in triplicate.

\subsection{In Vitro Antibacterial and Antifungal Properties of bLF-PLLA Membranes}

Antibacterial assays were performed by the agar diffusion method with cultures of Pseudomonas aeruginosa ATCC 10145 and Staphylococcus aureus ATCC 6538 grown in LB medium (yeast extract 5 g. $\mathrm{L}^{-1}$, sodium chloride 5 g.L $\mathrm{L}^{-1}$, Tryptone 10 g. $\mathrm{L}^{-1}$ ). Overnight cultures of the testing microorganisms were diluted in LB-Agar $(0.8 \% \mathrm{w} / \mathrm{v})$ to a final density of $1 \times 10^{6} \mathrm{CFUs} / \mathrm{mL}$ and layered on LB Agar (1.5\% w/v) plates. Pre-sterilized bLF-PLLA disks $(\varnothing=5 \mathrm{~mm})$ were placed in contact with the plate top-layer surface and incubated overnight at $37{ }^{\circ} \mathrm{C}$. Controls were performed using cellulose diffusion disks (Oxoid) and Kanamycin disks (30 $\mu$ g, BD Biosciences) as negative $\left(\mathrm{C}^{-}\right)$and positive $\left(\mathrm{C}^{+}\right)$controls for 
growth inhibition, respectively. After the incubation period, the diameters of growth inhibitory zones were evaluated.

Antifungal activity against Aspergillus nidulans was assessed by a modified spore germination assay as described before ${ }^{[41]}$. Briefly, spores were collected with the help of a sterile toothpick and inoculated in complete potato dextrose agar medium (PDA, BD Difco $\left.^{\mathrm{TM}}\right)$. Square samples of bLF-PLLA with an area of $100 \mathrm{~mm}^{2}$ were placed over the spore inoculation sites and incubated for $72 \mathrm{~h}$ at $37^{\circ} \mathrm{C}$. Digital images were recorded with a Chemidoc XRS System (BioRad) and the mycelial growth was measured and analyzed by ImageJ software. Cellulose disks embedded with itraconazol $\left(25 \mathrm{mg} \cdot \mathrm{mL}^{-1}\right)$ were used as positive control for growth inhibition. Additional antifungal assays involved evaluating the ability of the bLF-PLLA membranes to inhibit spore germination by scanning electron microscopy (SEM). Spores of A. nidulans were inoculated in complete medium (CM, salt solution $20 \mathrm{~mL}$, vitamin solution $10 \mathrm{~mL}$, casamino acids $1 \mathrm{~g}$, yeast extract $1 \mathrm{~g}$, peptone $2 \mathrm{~g}$, glucose $10 \mathrm{~g}$, per liter at $\mathrm{pH}$ 6.8) and placed in contact with the bLF-PLLA membranes followed by incubation at $37{ }^{\circ} \mathrm{C}$ for $18 \mathrm{~h}$. For fixation, the samples were soaked in a glutaraldehyde solution in PBS (1 mL of $2.5 \% \mathrm{v} / \mathrm{v}$ in PBS) for $1 \mathrm{~h}$ at room temperature (RT), rinsed with $1 \mathrm{~mL}$ of distilled water and dehydrated by immersion for $30 \mathrm{~min}$ in a series of successive ethanol/water solutions (0.5 mL of 55, 70, 80, 90, 95 and $100 \% \mathrm{v} / \mathrm{v})$. Samples were dried at RT and coated with a thin Au/Pd layer and analyzed by scanning electron microscopy (SEM, NanoSEM - FEI Nova 200) with a $5.0 \mathrm{kV}$ voltage and a through-lens detector (TLD).

\subsection{Statistics and Data Analysis}

One-way analysis of variance (ANOVA) with Bonferroni's post-test was carried out to compare the means of different data sets within each experiment using GraphPad Prism 5 
software. A value of $p<0.05$ was considered statistically significant. All experiments were performed in triplicate.

\section{Results and Discussion}

\subsection{Fabrication and Characterization of the Electrospun Nanofiber Membranes}

Electrospinning processing parameters deeply influence the fiber morphology and properties of the membranes, including initial polymer solution (polymer concentration and molecular weight, solvent boiling point, electrical permittivity or dipole moment), control of jet formation and solvent evaporation kinetics (flow rate, temperature, moisture, needle inner diameter, applied electric field), and collecting procedure (random or aligned fibers) ${ }^{[42,43]}$. One of the requirements for the formation of uniform fibers is the formation of a stable Taylor cone so, initial electrospinning experiments involved the determination of the optimal parameters to process PLLA (Table S1). Optimal conditions were found by applying an electric field of $1.0 \mathrm{kV} \cdot \mathrm{cm}^{-1}$, a flow rate of $0.5 \mathrm{~mL} \cdot \mathrm{min}^{-1}$, a needle inner diameter of $0.5 \mathrm{~mm}$ and a polymer concentration of $10 \%(\mathrm{w} / \mathrm{v})$. Therefore, these conditions were kept constant throughout all the experiments to study the influence of the bLF amount on the fiber average diameter and size distribution.

Smooth and non-defective fibers (Figure 1) were obtained from the PLLA polymer solution under the electrospinning conditions stated in Table S1. When bovine lactoferrin was added to the polymer solution, the electrospinning parameters showed to be equally optimal with a stable jet identical to that of the pristine PLLA. For all samples, the produced fibers were randomly distributed and non-defective with a smooth surface. Addition of bLF to the polymer solution led to a decrease in mean fiber diameter and a narrower size distribution for the sample with $20 \mathrm{wt} \%$ of bLF, which is probably related to the increase in conductivity promoted by the filler protein (Figure 1). Despite the reduction in the average fiber diameter, 
the overall porosity (calculated by Equation 1) of the different samples was not significantly affected by the incorporation of bLF, reaching values of $79 \pm 3 \%, 78 \pm 3 \%$ and $80 \pm 4 \%$, for PLLA, $10 \%$ bLF and $20 \%$ bLF, respectively.

Wettability of the electrospun samples was measured through water contact angle and it was observed that neat PLLA had the strongest hydrophobic behavior with a WCA of $130 \pm 2^{\circ}$. This value was slightly reduced with the presence of bLF in the membranes, reaching a value of $122 \pm 1^{\circ}$ for the sample with $20 \mathrm{wt} \%$ of bLF (Figure 1d). Kim et al. ${ }^{[44]}$ also reported that the WCA of titanium substrate decreases when human milk LF is immobilized on its surface, due to the affinity of the water molecules with the amide groups present in the bLF structure. The infrared spectrum of pure PLLA, bLF and bLF-PLLA electrospun membranes is depicted in Figure 2. The characteristic absorption bands of PLLA were observed both for the pristine and for the composite membranes at around $955 \mathrm{~cm}^{-1}, 1183 \mathrm{~cm}^{-1}, 1455 \mathrm{~cm}^{-1}, 1757 \mathrm{~cm}^{-1}$ and $2945 \mathrm{~cm}^{-1}$, which are related to the amorphous phase ${ }^{[32]}$. The characteristic protein absorption bands of amide I (1700-1600 cm-1, $\mathrm{C}=\mathrm{O}$ stretching vibration) centered around $1641 \mathrm{~cm}^{-1}$ and of amide II (1600-1500 $\mathrm{cm}^{-1}$, N-H bending with contribution of C-N stretching vibrations) centered around $1529 \mathrm{~cm}^{-1}$ were observed for the pure bLF, although at a lower intensity, for the bLF-PLLA composites ${ }^{[45]}$. The lower intensity of the amide I and amide II bands observed for the bLF-PLLA composites is attributed to the low amount of bLF within the fibers. Nevertheless, normalization of truncated amide I and amide II band regions allowed to confirm the presence of these bands in the FTIR spectra (Figure 2b). By comparing the spectra, it is possible to observe the absence of shifts in peak positions or the presence of new vibrational modes in the composites, other than those attributed to the amide bands. This demonstrates that both PLLA and bLF do not suffer structural changes during electrospinning and that there are no chemical reactions between bLF and PLLA. 


\subsection{Thermal Properties}

During electrospinning, much of the solvent present in the stretched jet is evaporated between the needle tip and the grounded collector. The fast solvent evaporation kinetics often results in a metastable phase ${ }^{[46]}$. Differential scanning calorimetry (Figure 3) was performed in the PLLA and bLF-PLLA electrospun samples to infer the influence of the filler in the thermal properties of the matrix. It was observed that incorporation of $10 \mathrm{wt} \%$ of bLF does not significantly change the glass transition $\left(T_{g}=57^{\circ} \mathrm{C}\right)$ or the cold-crystallization temperature $\left(T_{c c}=86^{\circ} \mathrm{C}\right)$. However, for the sample with $20 \mathrm{wt} \%$ of bLF, the cold-crystallization process is suppressed by the denaturation of the bLF that occurs at temperatures between $52-89{ }^{\circ} \mathrm{C}$ [47]. The melting temperature of the polymer matrix was not affected by addition of bLF reaching its maximum at approximately $154{ }^{\circ} \mathrm{C}$ (table S2), which is in accordance to the melting values reported for PLLA ${ }^{[30,33,35]}$.

Thermogravimetric analysis was used to study the degradation kinetics of pure bLF, PLLA and bLF-PLLA nanocomposite membranes (Figure 4). The thermogram of pure bLF reveals an initial weight loss of about $8 \mathrm{wt} \%$ at temperatures up to $120^{\circ} \mathrm{C}$ and is attributed to the loss of moisture, likely due to sample handling and storage conditions, followed by a strong mass loss at temperatures above $200{ }^{\circ} \mathrm{C}$ attributed to the decomposition of the protein ${ }^{[48]}$. Regarding the PLLA matrix, the thermogram does not present any dehydration process, which agrees with the hydrophobic behavior found through WCA (Figure 1d), and is dominated by a single stage of major weight loss between 300 and $400{ }^{\circ} \mathrm{C}$, attributed to the thermal decomposition of the polymer. When bLF was added to the PLLA electrospun fibers, the absence of adsorbed water was noticed for all nanocomposite samples, suggesting that the filler is randomly distributed throughout the core of the PLLA fibers, rather than be concentrated on the surface of the polymer fibers; this is in accordance to the results reported in Figure 1d, where the WCA did not decrease significantly with the presence of the lactoferrin in the PLLA electrospun fibers. The presence of bLF in the polymeric electrospun 
fibers also led to a slight decrease of the thermal stability of the polymer matrix (table S3). Nevertheless, the residual mass increases with the amount of the filler added to the polymeric fiber matrix.

The kinetics of the mass loss were studied through the analysis of the TGA experiments and by applying the Kissinger mathematical model ${ }^{[49]}$ :

$$
\ln \left(\frac{\beta}{T_{p}^{2}}\right)=\frac{\ln \left(A E_{a c t}\right)}{T_{p}}+\ln \left[n\left(1-\alpha_{p}\right)^{n}\right]-\frac{E_{a c t}}{R T_{p}}
$$

where, $\beta$ is the experimental heating rate, $T_{p}$ and $\alpha_{p}$ are the absolute temperature and the conversion at the maximum mass loss rate, and $E_{a c t}$ and $R$, are the thermal decomposition activation energy and the ideal gas constant, respectively.

According to the Kissinger's model for the degradation kinetics, the slope of the dot lines is proportional to the $E_{\text {act }}$ (Figure 4c). When bLF is added to the PLLA fiber matrix, there is an increase of the thermal decomposition activation energy to values close to those calculated for pure free bLF (Figure 4d).

\subsection{In Vitro Release of bLF}

The hydrolytic degradation and release profiles of PLLA and $20 \mathrm{wt} \%$ bLF-PLLA composites was performed in a PBS solution at $37^{\circ} \mathrm{C}$ (Figure 5). Hydrolytic degradation of PLLA is a slow process and almost no mass loss was observed for the neat PLLA membrane over 7 weeks of experiment, while a decrease in sample weight of $2.7 \pm 0.5 \%$ was detected for the bLF-PLLA membranes, suggesting that only a small amount of bLF is present on the surface of the PLLA electrospun fibers, while the main amount is dispersed in the core of the polymeric fiber (Figure 5a). 
During the in vitro degradation assays, the release of the bLF was also monitored (Figure 5b). The electrospun composites showed an initial release of bLF of $8.0 \pm 4.5 \%$ during the first 7 days, followed by a gradual release over $\sim 7$ weeks, of up to $17.7 \pm 4.4 \%$. The release profile was fitted with the Korsmeyer-Peppas model (Figure 5b), and a release exponent of $n=0.51$ was calculated, suggesting that the release of the bLF through the PLLA fibers is governed by an anomalous transport ${ }^{[39,40,50]}$, which was defined as a superposition of two apparently independent mechanisms of drug transport, a Fickian diffusion process and a case-II transport ${ }^{[40]}$. Accordingly, the initial boost of the bLF release to the PBS medium is due to the presence of the filler on the surface of the PLLA fiber, as it can be deduced from the evolution of mass loss (Figure 5a), while the gradual release after week 7 is possibly due to swelling of the PLLA promoted by diffusion of the PBS solution through the core of the polymeric fiber, allowing a steady release of bLF.

\subsection{Cytotoxicity Assays}

Cell viability of human skin fibroblasts (BJ-5ta cell line) in response to the bLF-PLLA composite membranes was assessed by indirect contact for 24 and $72 \mathrm{~h}$ using the MTS assay (Figure 6). After $24 \mathrm{~h}$ of incubation, no statistically significant differences were observed between the control $(100.0 \pm 2.8 \%)$ and $0 \mathrm{wt} \%$ bLF-PLLA $(92.7 \pm 12.5 \%)$ or $10 \mathrm{wt} \%$ bLFPLLA (102.8 $\pm 4.7 \%)$. Interestingly, the $20 \mathrm{wt} \%$ bLF-PLLA composite could induce a slight but significant proliferative effect in BJ-5ta cells, showing values of $109.3 \pm 6.2 \%$ of cell viability. Similarly, after 72 h of incubation, no significant cytotoxicity was observed for the produced materials, apart from the $20 \mathrm{wt} \%$ bLF-PLA composite which lead to a slight decrease in cell proliferation to about $88.9 \pm 8.8 \%$. Overall, these results confirm the cytocompatibility of the developed composite membranes, a key pre-requisite when developing biomedical devices. 


\subsection{In Vitro Antimicrobial Properties of bLF-PLLA Membranes}

The antibacterial activity of the electrospun bLF-PLLA membranes was assessed for $P$. aeruginosa and S. aureus by agar disk-diffusion method (Figure 7a). In opposition to the positive control and similarly to the PLLA electrospun fibers ( $0 \mathrm{wt} \%$ bLF-PLLA), no significant inhibition halos were found for either bacteria. As the occurrence of fungal infections in human skin and mucosa is growing, it fuels a growing demand for new antifungal strategies. Therefore, the antimicrobial studies were further extended to filamentous fungi by assessing the ability of the composite membranes to inhibit spore germination (Figure 7b) and mycelial growth (Figure 7c) of A. nidulans. Evaluation of the sporicidal activity was conducted by placing spores of A. nidulans in direct contact with the electrospun fibers, followed by SEM analysis. SEM micrographs reveal that inhibition of spore germination was dependent of lactoferrin content, with complete inhibition observed with the $20 \mathrm{wt} \%$ composite (Figure 7b). Indeed, while complete germination was observed with the PLLA sample (0 wt\% bLF-PLLA), this process showed to be limited or even absent in the samples containing $10 \mathrm{wt} \%$ and $20 \mathrm{wt} \% \mathrm{bLF}$, respectively. Considering these results, the ability of the composites to inhibit mycelial growth was tested using $20 \mathrm{wt} \%$ bLF-PLLA as a representative sample (Figure 7c). Although displaying a modest activity, the electrospun membrane could significantly inhibit mycelium growth when compared to the PLLA sample (0 wt\% bLF-PLLA) or to the control (direct inoculation with no sample) (Figure 7c).

The antimicrobial effect of bLF has been widely reported for an extensive range of pathogens, including those studied in this work ${ }^{[51]}{ }^{[52]}$ and ${ }^{[22]}$ (Aspergillus spp.). The lack of antibacterial activity observed in the disk diffusion assay and even the modest inhibition of mycelium growth may be explained by the slow release rate of bLF from the membranes (Figure 5b). For instance, only about $8 \%$ is released after 7 days of immersion in PBS. 
Furthermore, considering that water availability is considerable lower in the agar plate than in the in vitro release assay, then bLF concentration was probably negligible during the time of the experiment. This is further supported by the spore germination assay in which spores of $A$. nidulans were placed in direct contact with the fibers and thus exposed to a higher amount of bLF. Future strategies to overcome these limitations include the development of composites with higher bLF content and fibers' surface decoration with bLF after plasma treatment. Nevertheless, the positive results regarding the antifungal activity reinforce the potential use of these composites in biomedical applications, such as wound dressings.

\section{Conclusions}

This work reports the successful immobilization of a bioactive protein - bovine lactoferrin in PLLA fiber membranes produced by electrospinning. The immobilization of bLF resulted in a decrease of the average fiber diameter from $717 \pm 197 \mathrm{~nm}$ for pristine PLLA, down to $495 \pm 127 \mathrm{~nm}$ for the sample with $20 \mathrm{wt} \% \mathrm{bLF}$. Moreover, a decrease in wettability was also noticed for the nanocomposite membranes. The produced bLF-PLLA membranes did not induce cytotoxicity on human fibroblasts and noteworthy, the $20 \mathrm{wt} \%$ bLF-PLLA membrane was even able to induce cell proliferation after $24 \mathrm{~h}$ of indirect contact. Although not displaying a relevant antimicrobial activity against the bacteria tested, the composite membranes showed to be highly effective against the filamentous fungi A. nidulans. Indeed, the bLF-PLLA membranes showed to exert a strong sporicidal activity, as well as mycelium growth inhibition, against $A$. nidulans. Overall, the obtained results strongly support the application of these membranes for biomedical applications especially, for fungal infections management.

\section{Supporting Information}

Supporting Information is available from the Wiley Online Library or from the author 
Acknowledgements: This work was financed by the strategic programme UID/BIA/04050/2013 (POCI-01-0145-FEDER-007569) funded by national funds through Fundação para a Ciência e a Tecnologia (FCT) and by the ERDF through the COMPETE2020 - Programa Operacional Competitividade e Internacionalização (POCI). This article is a result of the project EcoAgriFood (NORTE-01-0145-FEDER-000009), supported by Norte Portugal Regional Operational Programme (NORTE 2020), under the PORTUGAL 2020 Partnership Agreement, through the European Regional Development Fund (ERDF). The present work was also supported by FCT within the ERA-NET IB, project FunBioPlas with grant number ERA-IB-15-089 and FCT reference ERA-IB-2-6/0004/2014. VS thank support from the COST Action MP1301 “New Generation Biomimetic and Customized Implants for Bone Engineering”.

Received: Month XX, XXXX; Revised: Month XX, XXXX; Published online: DOI: 10.1002/marc.((insert number)) ((or ppap., mabi., macp., mame., mren., mats.))

Keywords: lactoferrin, poly(lactic acid), antimicrobial activity, nanofiber membranes, electrospinning

[1] J. M. A. Blair, M. A. Webber, A. J. Baylay, D. O. Ogbolu, L. J. V. Piddock, Nat Rev Micro 2015, 13, 42.

[2] M. Ip, S. L. Lui, V. K. M. Poon, I. Lung, A. Burd, Journal of Medical Microbiology 2006, 55, 59.

[3] B. A. Lipsky, C. Hoey, Clinical Infectious Diseases 2009, 49, 1541.

[4] J. J. Castellano, S. M. Shafii, F. Ko, G. Donate, T. E. Wright, R. J. Mannari, W. G. Payne, D. J. Smith, M. C. Robson, International Wound Journal 2007, 4, 114. 
[5] J. S. Kim, E. Kuk, K. N. Yu, J.-H. Kim, S. J. Park, H. J. Lee, S. H. Kim, Y. K. Park, Y. H. Park, C.-Y. Hwang, Y.-K. Kim, Y.-S. Lee, D. H. Jeong, M.-H. Cho, Nanomedicine: Nanotechnology, Biology and Medicine 2007, 3, 95.

[6] W. K. Jung, H. C. Koo, K. W. Kim, S. Shin, S. H. Kim, Y. H. Park, Applied and Environmental Microbiology 2008, 74, 2171.

[7] M. L. W. Knetsch, L. H. Koole, Polymers 2011, 3, 340.

[8] M. Rai, A. Yadav, A. Gade, Biotechnology Advances 2009, 27, 76.

[9] S. M. Hussain, K. L. Hess, J. M. Gearhart, K. T. Geiss, J. J. Schlager, Toxicology in Vitro 2005, 19, 975 .

[10] L. Braydich-Stolle, S. Hussain, J. J. Schlager, M.-C. Hofmann, Toxicological Sciences 2005, 88, 412.

[11] A. Burd, C. H. Kwok, S. C. Hung, H. S. Chan, H. Gu, W. K. Lam, L. Huang, Wound Repair and Regeneration 2007, 15, 94.

[12] O. Levy, Blood 2000, 96, 2664.

[13] H. Wakabayashi, K. Yamauchi, M. Takase, Int Dairy J 2006, 16, 1241.

[14] S. Farnaud, R. W. Evans, Molecular Immunology 2003, 40, 395.

[15] I. A. García-Montoya, T. S. Cendón, S. Arévalo-Gallegos, Q. Rascón-Cruz, Biochimica et Biophysica Acta (BBA) - General Subjects 2012, 1820, 226.

[16] S. A. González-Chávez, S. Arévalo-Gallegos, Q. Rascón-Cruz, International Journal of Antimicrobial Agents 2009, 33, 301.e1.

[17] S. Hammerschmidt, G. Bethe, P. H. Remane, G. S. Chhatwal, Infection and Immunity 1999, 67, 1683.

[18] R. S. Bhimani, Y. Vendrov, P. Furmanski, Journal of Applied Microbiology 1999, 86, 135.

[19] F. Berlutti, C. Morea, A. Battistoni, S. Sarli, P. Cipriani, F. Superti, M. G. Ammendolia, P. Valenti, International Journal of Immunopathology and Pharmacology 2005, 18, 661. 
[20] M. P. Rogan, C. C. Taggart, C. M. Greene, P. G. Murphy, S. J. O'Neill, N. G. McElvaney, The Journal of Infectious Diseases 2004, 190, 1245.

[21] N. Kondori, L. Baltzer, G. T. Dolphin, I. Mattsby-Baltzer, International Journal of Antimicrobial Agents 2011, 37, 51.

[22] K. A. Zarember, J. A. Sugui, Y. C. Chang, K. J. Kwon-Chung, J. I. Gallin, The Journal of Immunology 2007, 178, 6367.

[23] J. H. Andersen, H. Jenssen, K. Sandvik, T. J. Gutteberg, Journal of Medical Virology 2004, 74, 262.

[24] A. K. Marr, H. Jenssen, M. R. Moniri, R. E. W. Hancock, N. Panté, Biochimie 2009, 91, 160.

[25] N. León-Sicairos, M. Reyes-López, C. Ordaz-Pichardo, M. de la Garza, Biochemistry and Cell Biology 2006, 84, 327.

[26] H. Jenssen, R. E. W. Hancock, Biochimie 2009, 91, 19.

[27] Z.-M. Huang, Y. Z. Zhang, M. Kotaki, S. Ramakrishna, Composites Science and Technology 2003, 63, 2223.

[28] S. Agarwal, J. H. Wendorff, A. Greiner, Polymer 2008, 49, 5603.

[29] D. B. Khadka, D. T. Haynie, Nanomedicine: Nanotechnology, Biology and Medicine 2012, 8, 1242.

[30] H. Tsuji, "Poly(Lactic Acid)", in Bio-Based Plastics, John Wiley \& Sons Ltd, 2013, p. 171.

[31] A. J. R. Lasprilla, G. A. R. Martinez, B. H. Lunelli, A. L. Jardini, R. M. Filho, Biotechnology Advances 2012, 30, 321.

[32] R. Clarisse, S. Vitor, C. Carlos Miguel, R. José Luís Gómez, L.-M. Senentxu, Science and Technology of Advanced Materials 2011, 12, 015001.

[33] V. Sencadas, C. M. Costa, G. Botelho, C. Caparrós, C. Ribeiro, J. L. Gómez-Ribelles, S. Lanceros-Mendez, Journal of Macromolecular Science, Part B 2012, 51, 411. 
[34] A. C. Areias, C. Ribeiro, V. Sencadas, N. Garcia-Giralt, A. Diez-Perez, J. L. Gomez Ribelles, S. Lanceros-Mendez, Soft Matter 2012, 8, 5818.

[35] T. A. M. Valente, D. M. Silva, P. S. Gomes, M. H. Fernandes, J. D. Santos, V. Sencadas, ACS Applied Materials \& Interfaces 2016, 8, 3241.

[36] W. S. Rasband, "ImageJ, U. S. National Institutes of Health, Bethesda, Maryland, USA, http://imagej.nih.gov/ij/", 1997-2011.

[37] D. Santos, D. M. Silva, P. S. Gomes, M. H. Fernandes, J. D. Santos, V. Sencadas, Journal of Colloid and Interface Science 2017, 504, 101.

[38] Y. Yan, V. Sencadas, J. Zhang, D. Wei, Z. Jiang, Advanced Materials Interfaces 2017, $\mathrm{n} / \mathrm{a}$.

[39] J. Siepmann, N. A. Peppas, Advanced Drug Delivery Reviews 2001, 48, 139.

[40] P. L. Ritger, N. A. Peppas, Journal of Controlled Release 1987, 5, 37.

[41] A. da Costa, R. Machado, A. Ribeiro, T. Collins, V. Thiagarajan, M. T. Neves-Petersen, J. C. Rodríguez-Cabello, A. C. Gomes, M. Casal, Biomacromolecules 2015, 16, 625.

[42] C. Ribeiro, V. Sencadas, J. L. G. Ribelles, S. Lanceros-Méndez, Soft Materials 2010, 8, 274.

[43] Seeram Ramakrishna, Kazutoshi Fujihara, Wee-Eong Teo, T.-C Lim , Z. M. . "An introduction to electrospinning and nanofibers", World Scientific Publishing Co. Pte. Ltd., Singapore, 2005.

[44] S. E. Kim, Y.-P. Yun, J. Y. Lee, K. Park, D. H. Suh, Colloids and Surfaces B: Biointerfaces 2014, 123, 191.

[45] X. Yao, C. Bunt, J. Cornish, S.-Y. Quek, J. Wen, Chemical Biology \& Drug Design 2014, 83, 560 .

[46] H. Zhou, T. B. Green, Y. L. Joo, Polymer 2006, 47, 7497.

[47] M. Iafisco, I. Foltran, M. Di Foggia, S. Bonora, N. Roveri, Journal of Thermal Analysis and Calorimetry 2011, 103, 41. 
[48] B. Wang, Y. P. Timilsena, E. Blanch, B. Adhikari, Drying Technology 2016, null.

[49] H. E. Kissinger, Journal of Research of the National Bureau of Standards 1956, 57, 217.

[50] S. Zuleger, B. C. Lippold, International Journal of Pharmaceutics 2001, 217, 139.

[51] P. K. Singh, M. R. Parsek, E. P. Greenberg, M. J. Welsh, Nature 2002, 417, 552.

[52] A. Aguila, A. G. Herrera, D. Morrison, B. Cosgrove, A. Perojo, I. Montesinos, J. Pérez,

G. Sierra, C. G. Gemmell, J. H. Brock, FEMS Immunology \& Medical Microbiology 2001, 31, 145.
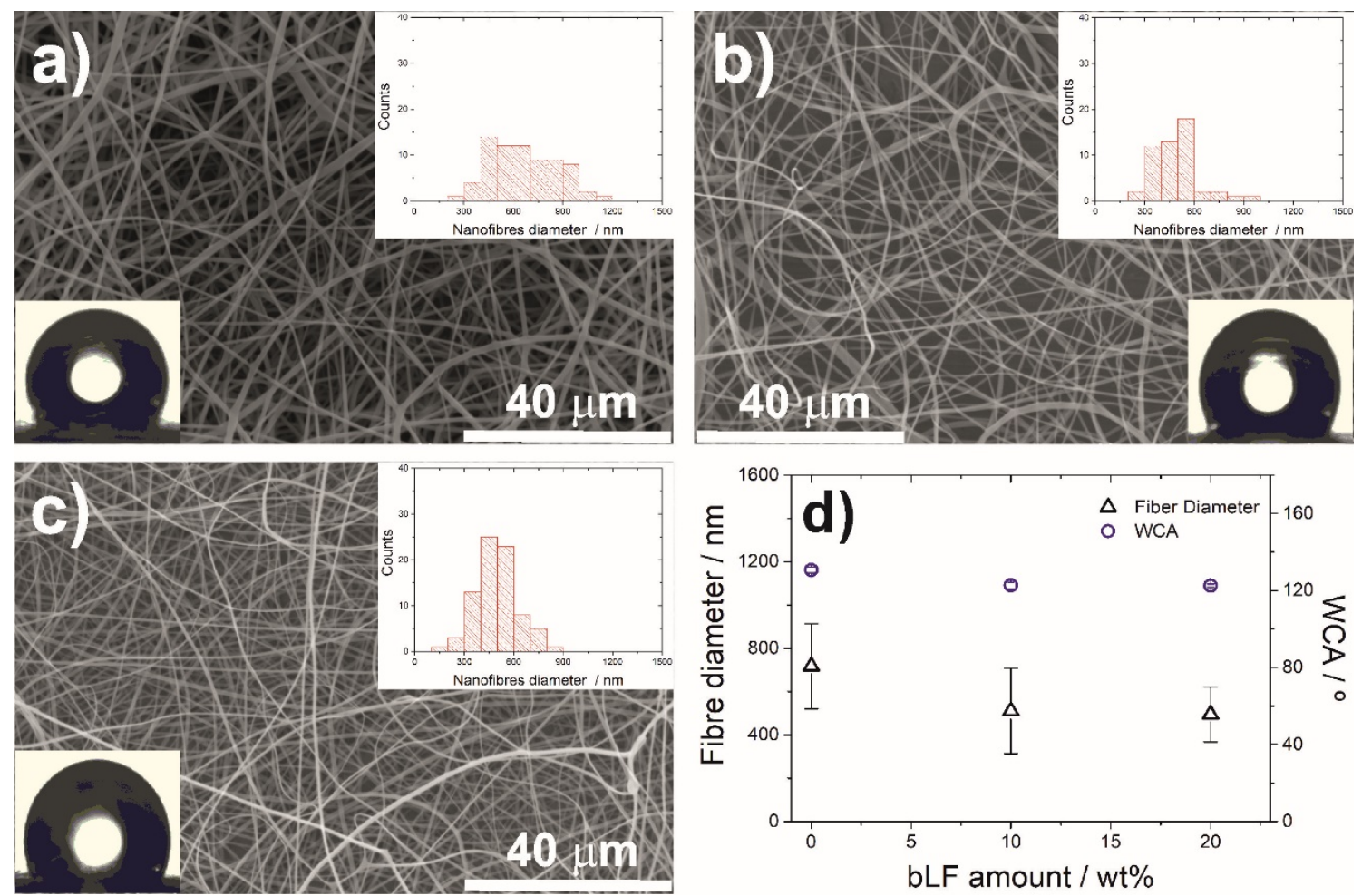

Figure 1. Morphology of bLF-PLA electrospun membranes with different bLF content: a) 0 $\mathrm{wt} \%$, b) $10 \mathrm{wt} \%$, c) $20 \mathrm{wt} \%$, and d) average fibre diameter and water contact angle (WCA). 

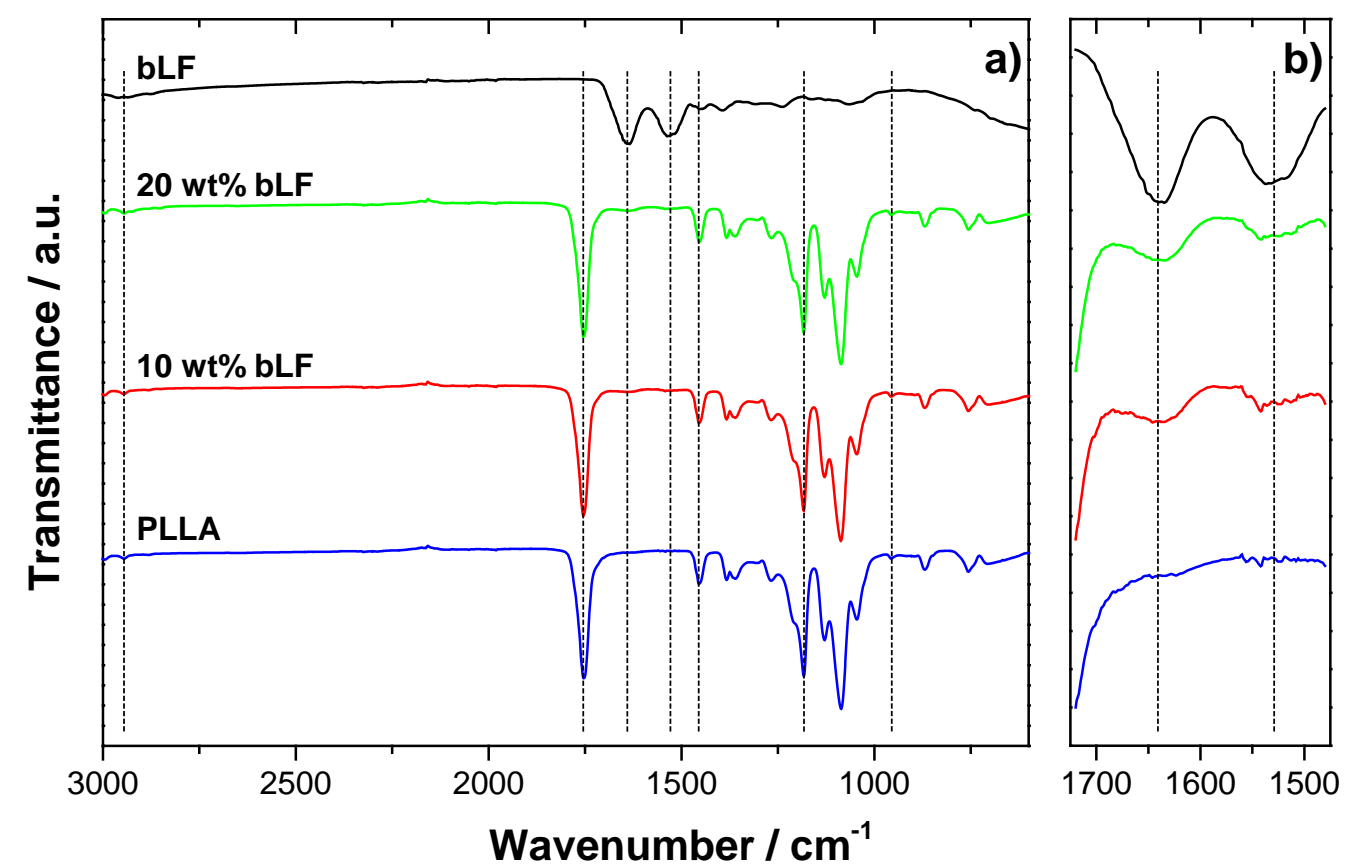

Figure 2. a) Fourier transformed infrared spectra of PLLA, bLF and bLF-PLLA electrospun membranes with 10 and $20 \mathrm{wt} \%$ of bLF, b) normalized spectra in the amide I (1700-1600 $\mathrm{cm}^{-}$ ${ }^{1}$ ) and amide II (1500-1400 $\mathrm{cm}^{-1}$ ) band regions. 


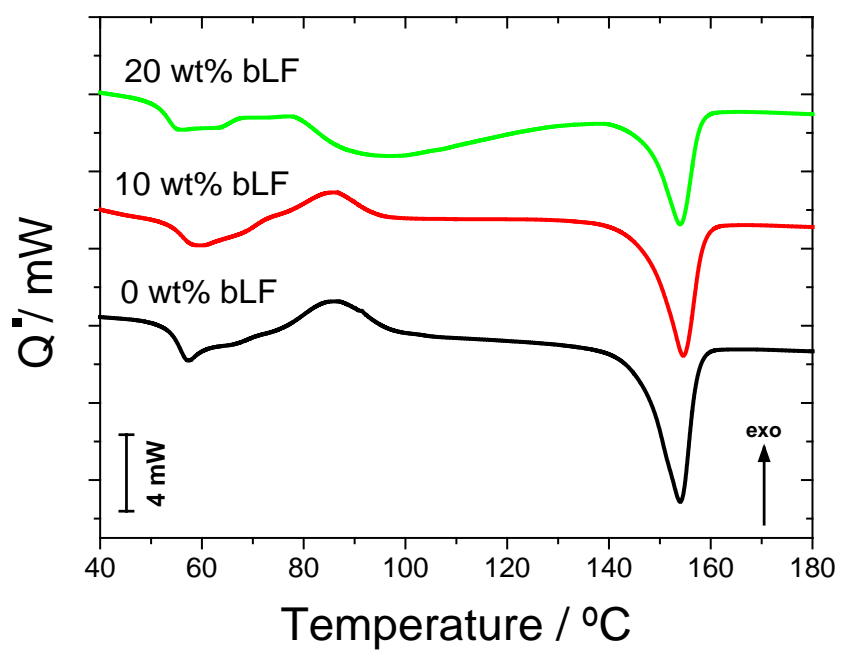

Figure 3. Differential scanning calorimetry of bLF-PLLA electrospun membranes. 

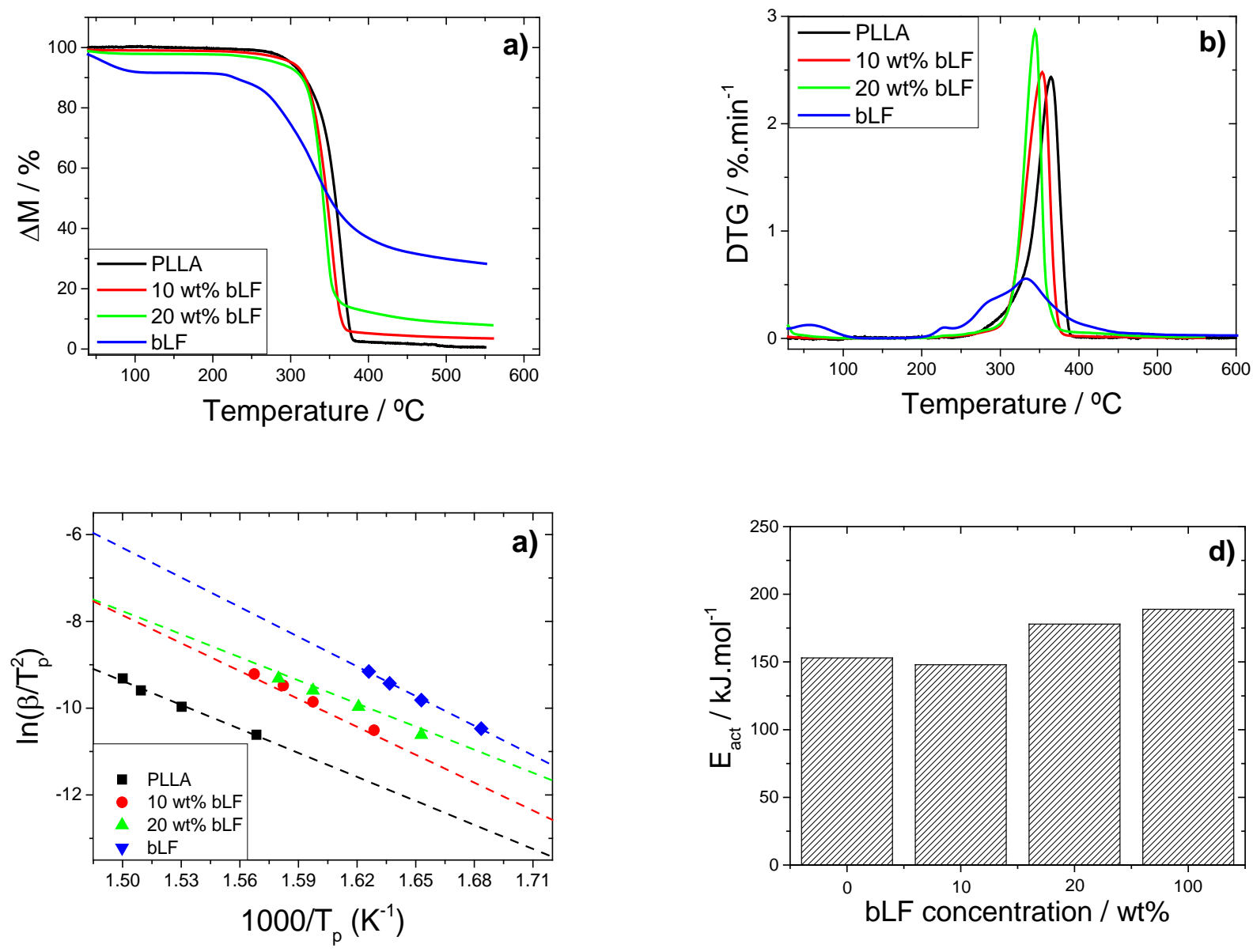

Figure 4. a) Thermal degradation profile of bLF-PLLA samples recorded at $20{ }^{\circ} \mathrm{C} \cdot \mathrm{min}^{-1}$, b) derivative of the thermal degradation process (heating rate $20^{\circ} \mathrm{C} \cdot \mathrm{min}^{-1}$ ), c) plots of the activation energy of the degradation process according to Kissinger model, and d) evolution of the activation energy with the concentration of the bLF in the different bLF-PLLA samples. 

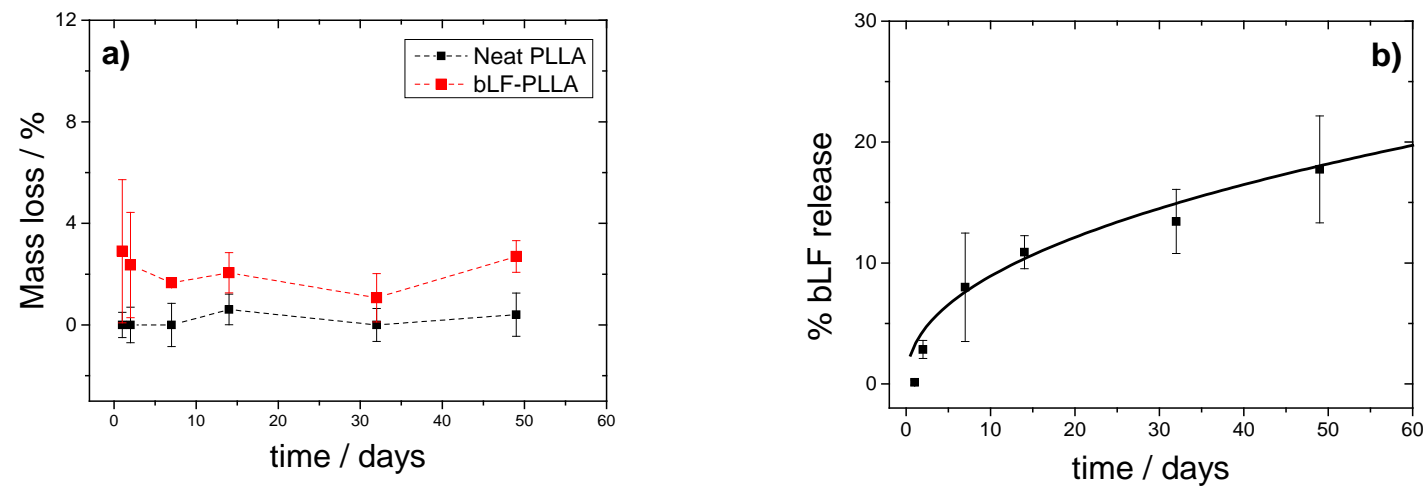

Figure 5. Hydrolytic degradation of electrospun composites (20 wt\%) in PBS (pH 7.4) at 37 ${ }^{\circ} \mathrm{C}$ (a); release profile of bLF and linear fitting using Korsmeyer-Peppas model (b). 


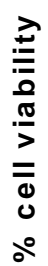

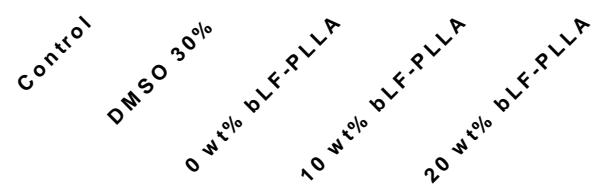

Figure 6. Cytotoxicity evaluation of bLF-PLLA electrospun membranes with different bLF contents (0 wt\%, $10 \mathrm{wt} \%$, and $20 \mathrm{wt} \%)$. Indirect contact assay was performed on normal human skin fibroblasts (BJ-5ta cell line) using the MTS assay and represented as \% cell viability in relation to the control. Bars represent mean \pm SD (ns, nonsignificant; ${ }^{*} p<0.05$, $* * p<0.01$ and $* * * * p<0.0001)$. 
a)

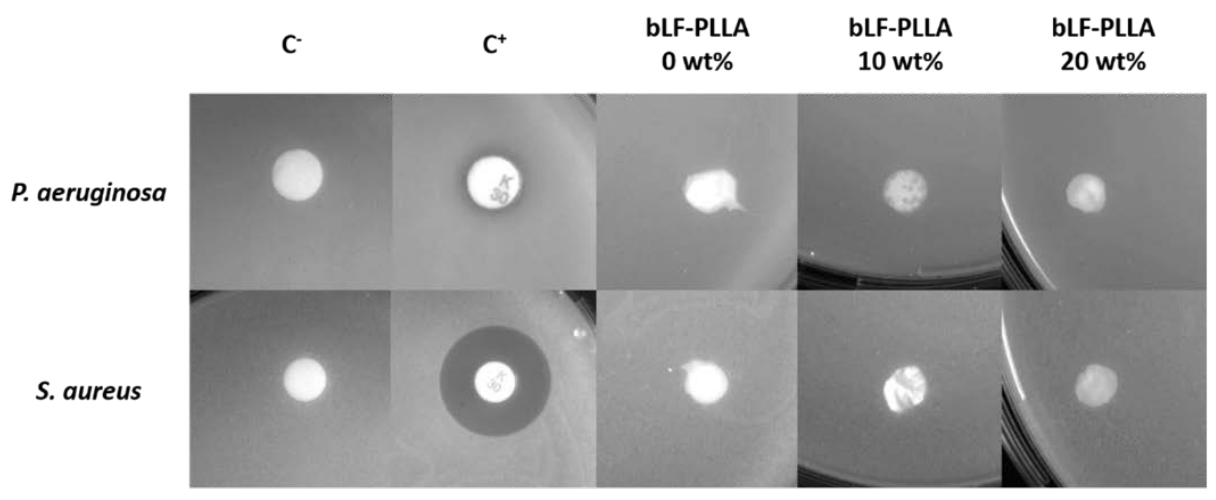

b)

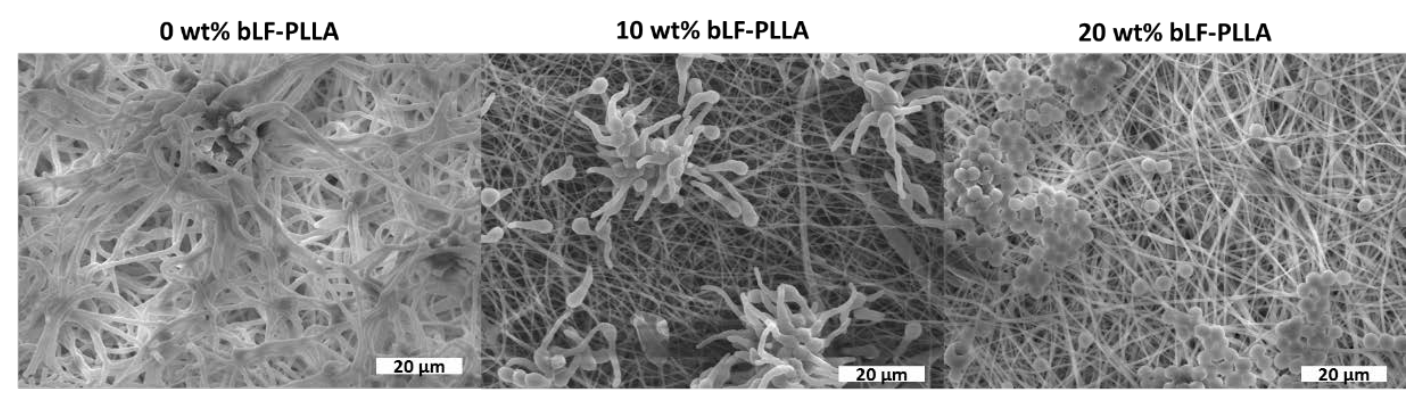

c)
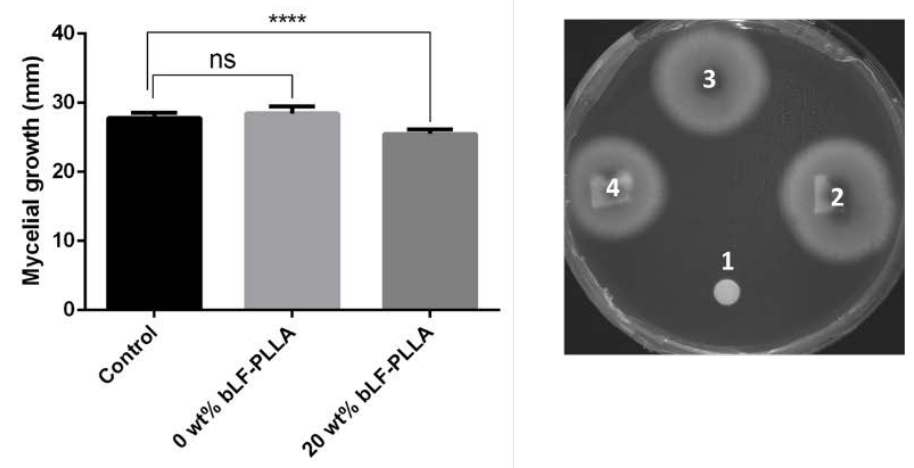

Figure 7. Antimicrobial activity of bLF-PLLA electrospun membranes ( $0 \mathrm{wt} \%, 10 \mathrm{wt} \%$, and $20 \mathrm{wt} \%$. a) Disk diffusion assay against $P$. aeruginosa and $S$. aureus in an agar layer for $18 \mathrm{~h}$ at $37^{\circ} \mathrm{C}$. b) SEM micrographs of germinating spores of $A$. nidulans in contact with the electrospun membranes for $18 \mathrm{~h}$ at $37^{\circ} \mathrm{C}$; scale bar: $20 \mu \mathrm{m}$. c) A. nidulans mycelial growth in contact with (1) itraconazol impregnated disk (25 mg/mL), (2) $0 \mathrm{wt} \%$ bLF-PLLA, (3) no sample, and (4) $20 \mathrm{wt} \%$ bLF-PLLA; inoculation site without any sample was used as control for mycelium growth; bars represent means \pm SD (ns, nonsignificant, $* * * * p \leq 0.0001$; experiments were performed in triplicate. 
Bovine lactoferrin, an iron-biding glycoprotein displays a wide set of physiological roles including a strong antimicrobial activity. The development of antimicrobial membranes by incorporating bovine lactoferrin in a poly(lactic acid) matrix is reported. The membranes show a strong sporicidal activity and mycelium growth inhibition against the filamentous fungi A. nidulans. These results strongly support their application for fungal infections management.

Raul Machado*, André da Costa, Dina M. Silva, Andreia C. Gomes, Margarida Casal and Vitor Sencadas*

Antibacterial and antifungal activity of poly(lactic acid)-bovine lactoferrin nanofiber membranes

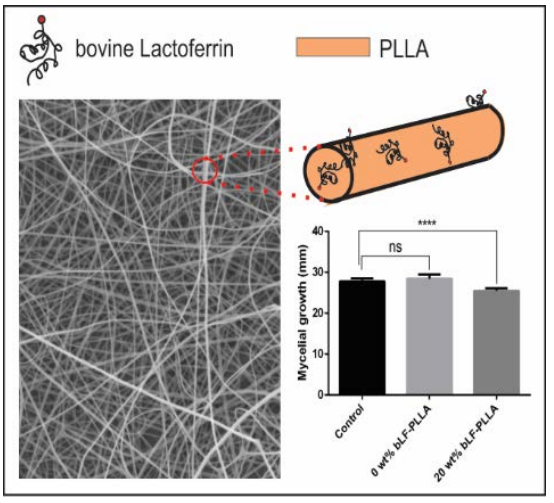


Copyright WILEY-VCH Verlag GmbH \& Co. KGaA, 69469 Weinheim, Germany, 2013.

\section{Supporting Information}

for Macromol. Biosci., DOI: 10.1002/mabi.2013\#\#\#\#\#

\section{Antibacterial and antifungal activity of poly(lactic acid)-bovine lactoferrin nanofiber membranes}

Raul Machado*, André da Costa, Dina M. Silva, Andreia C. Gomes, Margarida Casal and Vitor Sencadas*

Table S1. Electrospinning parameters used for the preparation of PLLA and bLF-PLLA membranes.

\begin{tabular}{cc}
\hline Parameters & Optimal conditions for electrospinning \\
\hline Applied electric field & $1.0 \mathrm{kV} \cdot \mathrm{cm}^{-1}$ \\
Flow rate & $0.5 \mathrm{~mL} \cdot \mathrm{min}^{-1}$ \\
Needle inner diameter & $0.5 \mathrm{~mm}$ \\
Polymer concentration & $10 \%(\mathrm{w} / \mathrm{v})$ \\
\hline
\end{tabular}

Table S2. Thermal properties and degree of crystallinity for the bLF-PLLA electrospun membranes.

\begin{tabular}{cccccc}
\hline $\begin{array}{c}\boldsymbol{b L F} \text { content } \\
(\boldsymbol{w t} \%)\end{array}$ & $\begin{array}{c}\boldsymbol{T}_{\boldsymbol{g}} \\
\left({ }^{\circ} \mathbf{C}\right)\end{array}$ & $\begin{array}{c}\boldsymbol{T}_{\boldsymbol{c c}} \\
\left(\boldsymbol{(}^{\circ} \mathbf{C}\right)\end{array}$ & $\begin{array}{c}\boldsymbol{T}_{\boldsymbol{m}} \\
\left({ }^{\circ} \mathbf{C}\right)\end{array}$ & $\begin{array}{c}\Delta \boldsymbol{H}_{\boldsymbol{m}} \\
\left(\mathbf{J . g}^{-\mathbf{1}}\right)\end{array}$ & $\begin{array}{c}\boldsymbol{C}_{\boldsymbol{p}} \\
\left(\mathbf{J . g}^{-\mathbf{1}} \cdot \boldsymbol{K}^{\mathbf{1}}\right)\end{array}$ \\
\hline 0 & 57 & 86 & 154 & 37 & 0.54 \\
10 & 57 & 86 & 154 & 31 & 0.44 \\
20 & 57 & $*$ & 154 & 28 & 0.40
\end{tabular}

* - suppressed by bLF; $T_{g}$ - glass transistion; $\mathrm{T}_{\mathrm{cc}}$ - cold-crystallization temperature; $T_{m}$ - melting temperature; $\Delta H_{m}-$ Melting enthalpy; $C_{p}$ - heat capacity at constant pressure. 
https://onlinelibrary.wiley.com/doi/pdf/10.1002/mabi.201700324

Table S3. Initial degradation, onset temperatures and activation energy of the bLF-PLLA electrospun samples.

\begin{tabular}{cccc}
\hline $\begin{array}{c}\boldsymbol{b L F} \text { content } \\
(\boldsymbol{w t} \%)\end{array}$ & $\begin{array}{c}\boldsymbol{T}_{\text {initial }} \\
\left({ }^{\circ} \mathbf{C}\right)\end{array}$ & $\begin{array}{c}\boldsymbol{T}_{\text {onset }} \\
\left({ }^{\circ} \mathbf{C}\right)\end{array}$ & $\begin{array}{c}\text { Residual mass at } 500^{\circ} \mathbf{C} \\
(\%)\end{array}$ \\
\hline 0 & 235 & 335 & 1 \\
10 & 223 & 327 & 4 \\
20 & 213 & 320 & 9 \\
100 & 201 & 269 & 30 \\
\hline
\end{tabular}

